\title{
$\angle$ Research Square \\ Phenotypic and Genotypic Characterization of the New Bacillus Cereus Phage SWEP1
}

\section{Chujin Ruan}

China Agricultural University

\section{Xinyao Niu}

China Agricultural University

\section{Guangzhou Xiong}

Huazhong University of Science and Technology

\section{GuoWei Chen}

Hefei University of Technology

Hanqing Wu

China Agricultural University

\section{Zechao Ma}

China Agricultural University

\section{Kun Zhu}

China Agricultural University

\section{Ying Liu}

Peking University

\section{Gang Wang ( $\sim$ gangwang@cau.edu.cn )}

China Agricultural University https://orcid.org/0000-0002-3201-6950

\section{Research Article}

Keywords: Bacillus cereus, Bacillus phage, Genome analysis

Posted Date: May 28th, 2021

DOl: https://doi.org/10.21203/rs.3.rs-522035/v1

License: (c) (1) This work is licensed under a Creative Commons Attribution 4.0 International License.

Read Full License 


\section{Abstract}

A new Bacillus cereus phage SWEP1 was isolated from black soil. The host lysis activity of phage SWEP1 has a relatively short latent time $(20 \mathrm{~min})$ and a small burst size of $83 \mathrm{PFU}$. The genome of SWEP1 consists of 162,461 bp with $37.77 \% \mathrm{G}+\mathrm{C}$ content. The phage encodes 278 predicted proteins where 103 were assigned functionally. No tRNA gene was found. Comparative genomics analysis indicated that SWEP1 is related to Bacillus phage B4 ( $86.91 \%$ identity, $90 \%$ query coverage). Phenotypic and genotypic characterization suggesting that SWEP1 is a new member of a new species in the genus Bequatrovirus, family Herelleviridae.

\section{Background}

Bacillus cereus, a ubiquitous bacterium in the environment, such as soil, water, air, food, and animal intestines [1]. It is a food-borne pathogen that produces enterotoxins and may cause severe food poisoning with symptoms such as nausea, vomiting, and diarrhea [2,3]. The discovery of new viruses targeting Bacillus cereus could contribute to a hypostatic understanding of virus-bacteria interactions, while also providing theoretical support for food safety, plant protection, and other fields.

Some bacteriophages capable of infecting $B$. cereus have been reported previously and are available in publicly available databases. The general features of some $B$. cereus bacteriophages are listed in Supplementary Table 1. The phages of $B$. cereus that have been isolated were extracted from soil [4-7], mud [8, 9], fermented foods [10, 11], lakes [12], and animal carcasses [13]. Genomic analysis of these phages has revealed that they all have a double-stranded DNA (dsDNA) genome with a G+C content between $34.0 \%$ and $41.7 \%[14,15]$. Their genome sizes span a wide range; TP21-L and VB_BceS_KLEB30$3 S$ are two temperate phages of $B$. cereus with small genome sizes $(37 \mathrm{~kb})[12,16]$ while most virulent phages have larger genome sizes $(153-166 \mathrm{~kb})[6,17]$.

In this study, a novel $B$. cereus bacteriophage SWEP1 was isolated from black soil samples in Northeast China $\left(43^{\circ} 18^{\prime} \mathrm{N}, 124^{\circ} 15^{\prime} \mathrm{E}\right)$ using $B$. cereus LB2 as the host bacterium, which was also isolated from the same soil sample. SWEP1 was characterized using transmission electron microscopy (TEM), one-step growth curve analysis tests, and stability tests. In addition, the whole genome of phage SWEP1 was completely sequenced and analyzed.

The host bacterium, designated strain Bacillus cereus LB2 (=CGMCC 1.18775), was isolated on LB agar medium [18] and taxonomically assigned based on the whole genome comparison. Average nucleotide identity (ANI) was determined to assess the genomic similarity between strain LB2 and B. cereus ATCC 14579 using the ANI calculator [19]. The ANI value between strain LB2 and ATCC 14579 was 96.5\%, which was higher than the $95 \%$ threshold of ANI relationship in species delimitation [20]. Therefore, the result confirms that the strain LB2 belongs to the Bacillus cereus species.

The phage was isolated from the same soil sample using the double-layer agar plating method followed by plaque purification [5]. Add $5 \mathrm{~g}$ of fresh soil to $200 \mathrm{~mL}$ of PBS buffer and shake at $160 \mathrm{rpm}, 25^{\circ} \mathrm{C}$ for 1 
h. The suspension soil was centrifuged at $3000 \times \mathrm{g}$ for $5 \mathrm{~min}$ to precipitate most soil particles. Three $\mathrm{ml}$ of supernatant was filtered with a $0.22 \mu \mathrm{m}$ filtration membrane (Syringe filter, Pall Corporation, USA) to remove microbial cells and part of soil particles. Then, mixed with $10 \mathrm{~mL}$ of a logarithmic growth culture of $B$. cereus $L B 2$ and incubated at $28^{\circ} \mathrm{C}$ for phage infection and replication. After $24 \mathrm{~h}$ incubation, the supernatant was filtered with a $0.22 \mu \mathrm{m}$ filtration membrane. To confirm the presence of bacteriophage in the supernatant, the sample was mixed with $B$. cereus using double-layer agar plates for overnight incubation. Some clear boundary plaques with a diameter ranging from 1.0 to $1.2 \mathrm{~mm}$ were detected (Fig. 1a). A single lytic plaque was selected and suspended in $1 \mathrm{~mL}$ of LB medium and the above purification procedure was repeated three times to obtain a pure phage preparation and named the phage SWEP1.

The morphological characteristics of the phage SWEP1 were obtained by TEM (JEM-1400 electron microscope) [19]. TEM analysis shows that SWEP1 has an icosahedral symmetry head (diameter of $72 \pm$ $2 \mathrm{~nm}$ ) and a long tail (157 $\pm 2 \mathrm{~nm}$ ) (Fig. 1b). All the Bacillus cereus phages isolated and studied to date have belonged to order Caudovirales (tailed phage), of which about $75 \%$ belong to Myoviridae [1-12]. Morphological comparisons of phage SWEP1 and other $B$. cereus phages shows that the head and tail sizes of phage SWEP1 are smaller than those of $B$. cereus phages B4 $(85 \mathrm{~nm}, 213 \mathrm{~nm})$ [3], B. cereus phages Bc431v3 $(85.4 \pm 3 \mathrm{~nm}, 180 \pm 3 \mathrm{~nm})$ [9] and $B$. cereus phages Flapjack $(91 \mathrm{~nm}, 232 \mathrm{~nm})$ [5], suggesting that phage SWEP1 is a novel species.

For detected one-step growth curve of phage SWEP1, the pure phage SWEP1 was mixed with logarithmic growth culture $B$. cereus LB2 $\left(\mathrm{OD}_{600}=1\right)$ at the ratio of 0.1 . The mixture was centrifuged at $8000 \times \mathrm{g}$ for 2 min to remove the unabsorbed phage. The precipitation was resuspended in a liquid medium and shaking incubated at $28^{\circ} \mathrm{C}$. One hundred $\mu \mathrm{L}$ aliquots of culture were then collected at selected time intervals to determine the viral abundance using the double-layer agar method. Latent period, lytic cycle, and burst size of SWEP1 were determined by constructing a one-step growth curve (Fig. 1c). The results showed that phage SWEP1 holds a latent period of $20 \mathrm{~min}$ and an outbreak period of 20 80 min. The burst size was about $83 \mathrm{PFU}$, calculated as the ratio of phage particles at the end of the outbreak to the beginning of the latent period. SWEP1 has a long latent period and a smaller burst size than B4 (15min, 200 PFU), which is another Myophage that has been studied to infect B. cereus ATCC 10876 [3]. Phage Bc431v3, which is also a Myophage that infects $B$. cereus strain LJH431, has a long latent period (85 \pm 5 min) and larger burst size (318 \pm 5 PFU) than SWEP1 [9].

The isolated phage was tested for its stability at different temperatures $\left(25^{\circ} \mathrm{C}, 40^{\circ} \mathrm{C}, 50^{\circ} \mathrm{C}\right.$, and $\left.60^{\circ} \mathrm{C}\right)$ using an initial phage abundance of $3.36 \times 10^{6} \mathrm{pfu} / \mathrm{mL}, 30 \mathrm{~min}$ incubation at $\mathrm{pH} 7$ [21]. Phages were additionally inoculated into LB medium under different $\mathrm{pH}$ conditions $(4,5,6,7,8,9,10,11)$ at an initial phage titter of $5.65 \times 10^{6} \mathrm{pfu} / \mathrm{mL}, 30 \mathrm{~min}$ incubation at $25^{\circ} \mathrm{C}$. After incubation, the phage abundance was determined. Phage stability tests found that phage SWEP1 could sustain a high titer at $25^{\circ} \mathrm{C}, 40^{\circ} \mathrm{C}$, and $50^{\circ} \mathrm{C}$ after incubation for $30 \mathrm{~min}$. SWEP1 is inactivated when temperatures reach $60^{\circ} \mathrm{C}$ (Fig. $1 \mathrm{~d}$ ). SWEP1 can sustain a high titer at $\mathrm{pH} 5 \sim 10$ after incubation for $30 \mathrm{~min}(P>0.05)$, and SWEP1 titer drops rapidly at 
$\mathrm{pH} 4$ and $\mathrm{pH} 11(P<0.05)$ (Fig. 1e). The stability tests demonstrated that phage SWEP1 is stable over a wide range of temperature and $\mathrm{pH}$ conditions.

The DNA of phage SWEP1 was extracted with a conventional phenol/chloroform/isoamyl alcohol method. The whole genome of SWEP1 was sequenced at the Illumina NovaSeq 6000 platform (Illumina, San Diego, CA, USA) with a paired-end read size of $150 \mathrm{bp}$. The raw reads (27887465) were quality controlled with FastQC and Trimmomatic. 93.82\% reads (26163099) were preserved after trimming. Reads that mapped to the host genome were removed for future analysis using BWA (v0.7.17) [22]. Finally, $88.21 \%$ clean reads (23077342) remained after quality control and host genome moving, then these reads were assembled using MEGAHIT (v1.2.9) [23]. The PHASTER online servers (http://phaster.ca/) and Prodigal were used to identify putative open reading frames (ORFs) and functional annotation [24]. The ORFs predict results were merged and checked manually. The ORFs predicted by Prodigal annotation was performed by BLASTN against the nonredundant nucleotide database (NR) with an E-value cutoff of $10^{-5}$. tRNAscan-SE was used to detect the presence of tRNA genes in the SWEP1 genome. Comparison of the SWEP1 genome with other similar phage genomes was performed by BLASTN. The complete genome sequence was submitted to the GenBank database under accession number MW787012. The genome of SWEP1 was assembled based on 23,077,342 cleaned paired-end reads and has a size of $162,461 \mathrm{bp}$ and a low $\mathrm{G}+\mathrm{C}$ content of $37.77 \%$. The genome of SWEP1 is a closed circular double-stranded DNA molecule with 278 predicted ORFs. A total of 142,627 nucleotides ( $87.82 \%$ of the genome) are involved in protein-coding. The average ORF length is $513 \mathrm{bp}$ with a range from 113 to $6056 \mathrm{bp} .175$ predicted ORFs (62.9\%) were predicted to encode hypothetical proteins whereas 103 were functionally annotated (Fig 2a). There is no tRNA gene detected in the SWEP1 genome. The result indicates that the protein synthesis of phage SWEP1 is completely reliant on the host bacteria tRNA. Genes annotation performed by PHASTER and Prodigal identify different functional clusters in the SWEP1 genome, including virion morphogenesis (head, tail, capsid, plate, portal), terminase, DNA manipulation, and modification (Table S3).

SWEP1 contains DNA replication and modification-related genes, including endonuclease- and exonuclease-encoding genes. Some gene products are predicted to be responsible for degrading the host genome and therefore the degrading products deoxyribonucleotides would be the raw material of phage DNA synthesis [25]. VSR endonuclease and recombination endonuclease subunit D12 were also found in this genome [26].

Some lysis regions were identified in the SWEP1 genome. The holin-lysin system plays an important role in the phage lytic cycle, as holin and cell wall hydrolases degrade the host-cell membrane to allow endolysins to reach the bacterial cell wall [27]. Terminase-coding genes were also identified that are essential for the phage genome packaging [28]. Additionally, the portal protein is an essential component of the prohead that is needed in the DNA translocation process [29]. It was predicted in the SWEP1 genome too, showing similarity to the Bac. B5S portal protein. 
We used the BLASTN online server to query the SWEP1 genome against the NCBI complete bacteriophages database (number of sequences: 4062) to identify similar phage genomes with a total score cutoff of 20000 (Table S2). The phylogenetic tree of SWEP1-related phages (top 10) based on whole-genome was constructed by ClustalW using default parameters [30]. iTol (https://itol.embl.de/) was applied to visualize the whole-genome-based phylogenetic tree. We searched the SWEP1 genome against the complete bacteriophage genomes database using the BLASTN online serve (https://blast.ncbi.nlm.nih.gov/Blast.cgi) with an E-value cutoff $10^{-5}[31,32]$. The most similar phage was Bacillus phage B4 (txid: 1141133), with high identity (86.91\%) and high query cover (90\%). MUSCLE (http://www.drive5.com/muscle/) [33] was used to construct a whole-genome-based phylogenetic tree of SWEP1-related viruses (Table S2). The accession number of the SWEP1-related phages used in the construction of the phylogenetic tree are listed on the tree (Fig 2b). The whole-genome-based phylogenetic tree illustrated that SWEP1 belongs to the genus Bequatrovirus and it was separate from the subcluster composed of Bacillus cereus phage B4 and B5S. Similar genomes based on genome identity and phylogenetic trees were used for the comparative genomic analysis. The result indicates many homologous genes of SWEP1 and similar genomes (Fig 2c). These results support that SWEP1 represents a new viral species and occur some recombination events during the evolution process.

In conclusion, we isolated SWEP1, a new phage from soils that can infect Bacillus cereus. The new phage was characterized based on its morphology, biological characteristics, genomic sequence, and phylogenetic relationships. Its short latent period and high stability suggest that phage SWEP1 has the potential as a candidate biocontrol agent against $B$. cereus for food safety. Based on genomic and phylogenetic analyses, SWEP1 can be considered a new member of a new species in the genus Bequatrovirus, family Herelleviridae, and most closely related to Bacillus phage B4 (NCBI: txid1141133). The GenBank accession number for phage SWEP1 is MW787012.

\section{Declarations}

\section{Availability of data and materials}

All data generated or analyzed during this study are included in this published article.

\section{Conflicts of interest/Competing interests}

The authors declare no conflict of interest.

\section{Funding}

This work was supported by the National Natural Science Foundation of China (41877412), project to Attract High Level Foreign Experts (G20190001094), the National Key R\&D Program of China (2016YFD0200306), and the 2115 Talent Development Program of China Agricultural University. 


\section{Authors' contributions}

CJR, GWC, and GW organized and designed experiments; CJR, XYN, HQW, and ZCM performed experiments. CJR, XYN, GZX analyzed data. CJR, XYN, GZX, YL wrote the first draft of the manuscript. All authors read and approved the final manuscript.

\section{Acknowledgments}

We thank David R. Johnson, Josep Ramoneda Massague, and Elyse Stachler for improving the quality and clarity of this manuscript.

\section{References}

1. Kim SK, Kim K-P, Jang SS, Shin EM, Kim M-J, Oh S, Ryu S (2009) Prevalence and toxigenic profiles of Bacillus cereus isolated from dried red peppers, rice, and Sunsik in Korea. J Food Prot 72(3):578-82. https://doi.org/10.4315/0362-028X-72.3.578.

2. Bottone EJ (2010) Bacillus cereus, a volatile human pathogen. Clin Microbiol Rev 23(2):382-398. https://doi.org/10.1128/CMR.00073-09.

3. Granum PE, Lund T (1997) Bacillus cereus and its food poisoning toxins. FEMS Microbiol Lett 157:223-228. https://doi.org/10.1111/j.1574-6968.1997.tb12776.x.

4. Klumpp J, Schmuki M, Sozhamannan S, Beyer W, Fouts DE, Bernbach V, Calendar R, Loessner M (2014) The odd one out: Bacillus ACT bacteriophage CP-51 exhibits unusual properties compared to related Spounavirinae W.Ph. and Bastille. Virology 462-463L:299-308. https://doi.org/10.1016/j.virol.2014.06.012.

5. Erill I, Caruso SM (2016) Genome sequence of Bacillus cereus group phage SalinJah. Genome Announc 4(5). https://doi.org/10.1128/genomeA.00953-16.

6. Erill I, Caruso SM (2017) Bacillus cereus group bacteriophage Flapjack genome sequence. Genome Announc 5(31):e00700-17. https://doi.org/10.1128/genomeA.00700-17.

7. Erill I, Caruso SM (2015) Complete genome sequence of Bacillus cereus group phage TsarBomba. Genome Announc 3(5). https://doi.org/10.1128/genomeA.01178-15

8. Lee JH, Shine H, Son B, Heu S, Ryu S (2013) Characterization and complete genome sequence of a virulent bacteriophage B4 infecting food-borne pathogenic Bacillus cereus. Arch Virol 158(10):21012108. https://doi.org/10.1007/s00705-013-1719-2.

9. El-Arabi TF, Griffiths MW, She YM, Villegas A, Lingohr EJ, Kropinski AM (2013) Genome sequence and analysis of a broad-host range lytic bacteriophage that infects the Bacillus cereus group. Virol $\mathrm{J}$ 10(1):48. https://doi.org/10.1186/1743-422X-10-48.

10. Shin H, Bandara N, Shin E, Ryu S, Kin K (2011) Prevalence of Bacillus cereus bacteriophages in fermented foods and characterization of phage JBP901. Res Microbiol 162(8):791-797. 
https://doi.org/10.1016/j.resmic.2011.07.001

11. Lee JH, Shin H, Son B, Ryu S (2012) Complete genome sequence of Bacillus cereus bacteriophage BCP78. J Virol 86(1):637-8. https://doi.org/10.1128/JVI.06520-11.

12. Monika S, Deividas T, Kotryna K, Rolandas M, Sigitas S, Eugenijus S (2020) Complete genome sequence of Bacillus cereus bacteriophage vB_BceS_KLEB30-3S. Microbiol Resour Announc 9(20). https://doi.org/10.1128/MRA.00348-20.

13. Beyer W, Bellan S, Eberle G, Ganz HH, Getz WM et al (2012) Distribution and molecular evolution of Bacillus anthracis genotypes in Namibia. PLoS Negl Trop Dis 6:e1534. https://doi.org/10.1371/journal.pntd.0001534.

14. Na HJ, Kong M, Ryu S (2016) Characterization of LysPBC4, a novel Bacillus cereus-specific endolysin of bacteriophage PBC4. FEMS Microbiol Lett 363(12). https://doi.org/10.1093/femsle/fnw092.

15. Kong M, Kim M, Ryu S (2012) Complete genome sequence of Bacillus cereus bacteriophage PBC1. J Virol 86(11):6379-80. https://doi.org/10.1128/JVI.00706-12.

16. Klumpp J, Calendar R, Loessner MJ (2010) Complete nucleotide sequence and molecular characterization of Bacillus phage TP21 and its relatedness to other phages with the same name. Viruses 2(4):961-971. https://doi.org/10.3390/v2040961.

17. Schuch R, Pelzek AJ, Fazzini MM, Nelson DC, Fischetti VA (2014) Complete genome sequence of Bacillus cereus Sensu Lato bacteriophage Bcp1. Genome Announc 2(3). https://doi.org/10.1128/genomeA.00334-14.

18. Sun C, Wang RJ, Su Y, Fu G, Zhao Z et al (2017) Hyphobacterium vulgare gen. nov., sp. nov., a novel alphaproteobacterium isolated from seawater. Int J Syst Evol Micr 67(5):1169-1176. https://doi.org/10.1099/ijsem.0.001780.

19. Yoon S-H, Ha SM, Lim J, Kwon S, Chun J (2017) A large-scale evaluation of algorithms to calculate average nucleotide identity. Antonie van Leeuwenhoek. 110:1281-1286. https://doi.org/10.1007/s10482-017-0844-4.

20. Richter M, Rosselló-Móra R. (2009) Shifting the genomic gold standard for the prokaryotic species definition. Proc Natl Acad Sci USA 106(45):19126-19131. https://doi.org/10.1073/pnas.0906412106.

21. Yuan X, Zhang S, Wang J, Li C, Na L et al (2021) Isolation and characterization of a novel Escherichia coli Kayfunavirus phage DY1. Virus Res 293:198274. https://doi.org/10.1186/s12985019-1241-6.

22. Li H, Durbin R (2009) Fast and accurate short read alignment with Burrows-Wheeler transform. Bioinformatics 25(14): 1754-60. https://doi.org/10.1093/bioinformatics/btp324.

23. Li D, Liu CM, Luo R Sadakane K, Lam TW (2015) MEGAHIT: an ultra-fast single-node solution for large and complex metagenomics assembly via succinct de Bruijn graph. Bioinformatics (Oxford, England). 31(10):1674-1676. https://doi.org/10.1093/bioinformatics/btv033.

24. Arndt D, Grant JR, Marcu A, Sajed T, Pon A, Liang Y, Wishart DS (2016) PHASTER: a better, faster version of the PHAST phage search tool. Nucleic Acids Res 44(W1):W16-W21. 
https://doi.org/10.1093/nar/gkw387.

25. Kropinski AM, Waddell T, Meng J, Franklin K, Ackermann HW, Ahmed R, Mazzocco A, Yates J, Lingohr EJ, Johnson R (2013) The host-range, genomics and proteomics of Escherichia coli 0157:H7 bacteriophage rV5. Virol J 10(1):76. https://doi.org/10.1186/1743-422X-10-76.

26. Bażlekowa-Karaban M, Adamczyk-Popławska M, Kwiatek A (2017) Characterization of Vsr endonucleases from Neisseria meningitidis. Microbiology (Reading, England) 163(7):1003-1015. https://doi.org/10.1099/mic.0.000492.

27. Chen X, Gao T, Peng Q, Zhang J, Chai Y, Song F (2018) Novel cell wall hydrolase cwlC from Bacillus thuringiensis is essential for mother cell lysis. Appl Environ Microbiol 84(7). https://doi.org/10.1128/AEM.02640-17.

28. Petrovski S, Seviour RJ, Tillett D (2011) Genome sequence and characterization of the Tsukamurella bacteriophage TPA2. Appl Environ Microbiol 77(4):1389-1398. https://doi.org/10.1128/AEM.0193810.

29. Lhuillier S, Gallopin M, Gilquin B, Brasilès S, Lancelot N et al (2009) Structure of bacteriophage SPP1 head-to-tail connection reveals mechanism for viral DNA gating. Proc Natl Acad Sci USA 106(21):8507-8512. https://doi.org/10.1073/pnas.0812407106.

30. Larkin MA, Blackshields G, Brown NP, Chenna R, McGettigan PA et al (2007) Clustal W and Clustal X version 2.0. Bioinformatics (Oxford, England) 23(21):2947-2948. https://doi.org/10.1093/bioinformatics/btm404.

31. Paez-Espino D, Eloe-Fadrosh EA, Pavlopoulos GA, Thomas AD, Huntemann M, Mikhailova N, Rubin E, Ivanova NN, Kyrpides NC (2016) Uncovering Earth's virome. Nat 536(7617):425-430. https://doi.org/10.1038/nature19094.

32. Paez-Espino D, Pavlopoulos GA, Ivanova NN, Kyrpides NC (2017) Nontargeted virus sequence discovery pipeline and virus clustering for metagenomic data. Nat Protoc 12(8):1673-1682. https://doi.org/10.1038/nprot.2017.063.

33. Edgar RC (2004) MUSCLE: multiple sequence alignment with high accuracy and high throughput. Nucleic Acids Res 32(5):1792-1797. https://doi.org/10.1093/nar/gkh340.

\section{Figures}


(a)

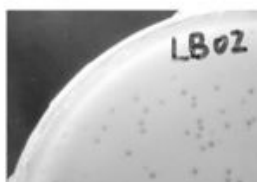

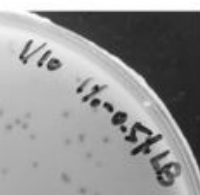

(b)
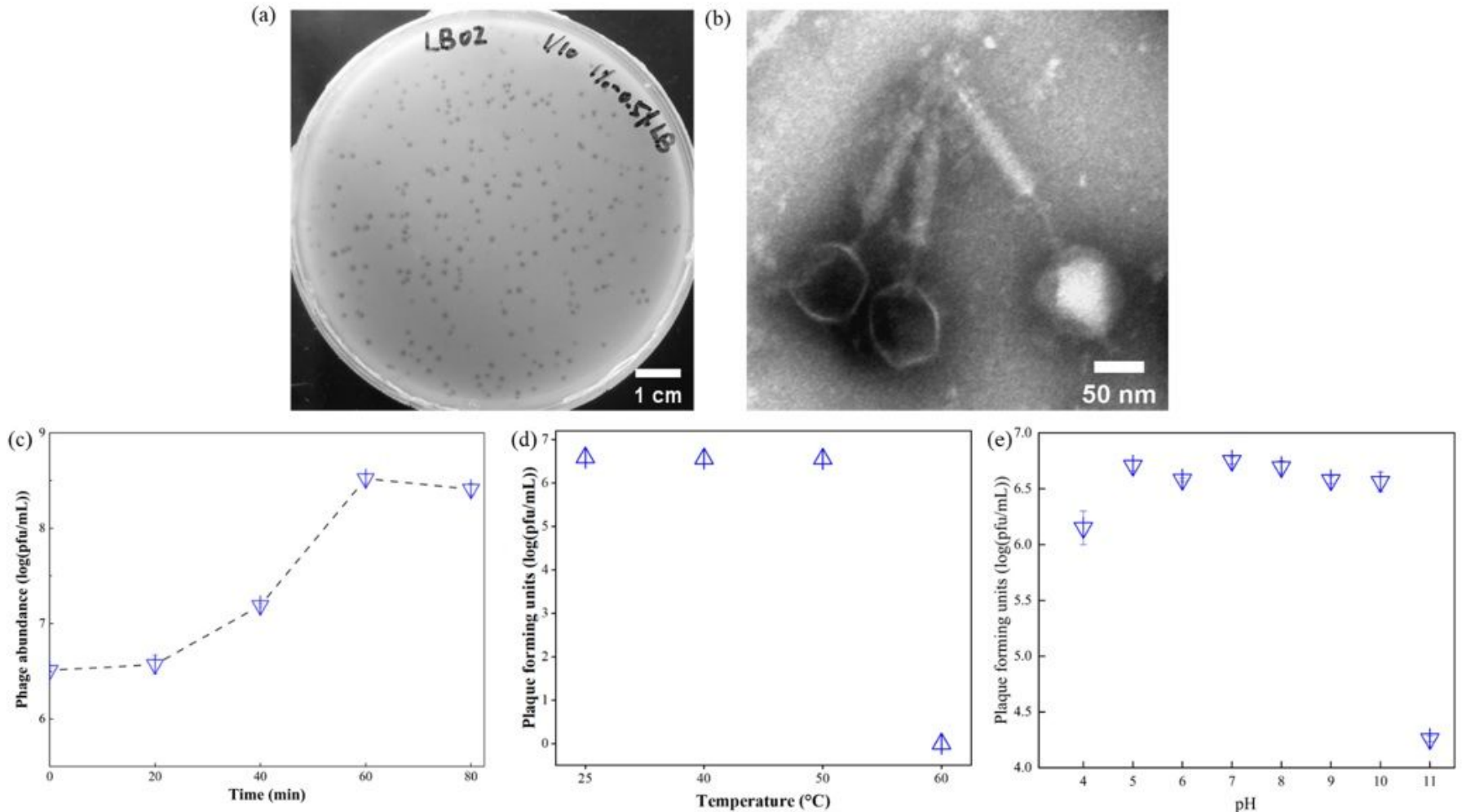

\section{Figure 1}

Morphological and physiological characteristics of phage SWEP1. (a) Plaques formed by SWEP1 on B. cereus strain LB2, scale bar is $1 \mathrm{~cm}$; (b) transmission electron microscopic image of SWEP1, scale bar is $50 \mathrm{~nm}$; (c) one-step growth curve of phage SWEP1; (d) the effects of incubation temperature on phage stability at $3.36 \times 106 \mathrm{pfu} / \mathrm{mL}$ initial phage titer estimated at 30 mins after inoculation at $\mathrm{pH}$ value of 7 ; and (e) the effects of $\mathrm{pH}$ value on phage stability at $5.65 \times 106 \mathrm{pfu} / \mathrm{mL}$ initial phage titer estimated at 30 mins after inoculation under $25^{\circ} \mathrm{C}$. 
(a)

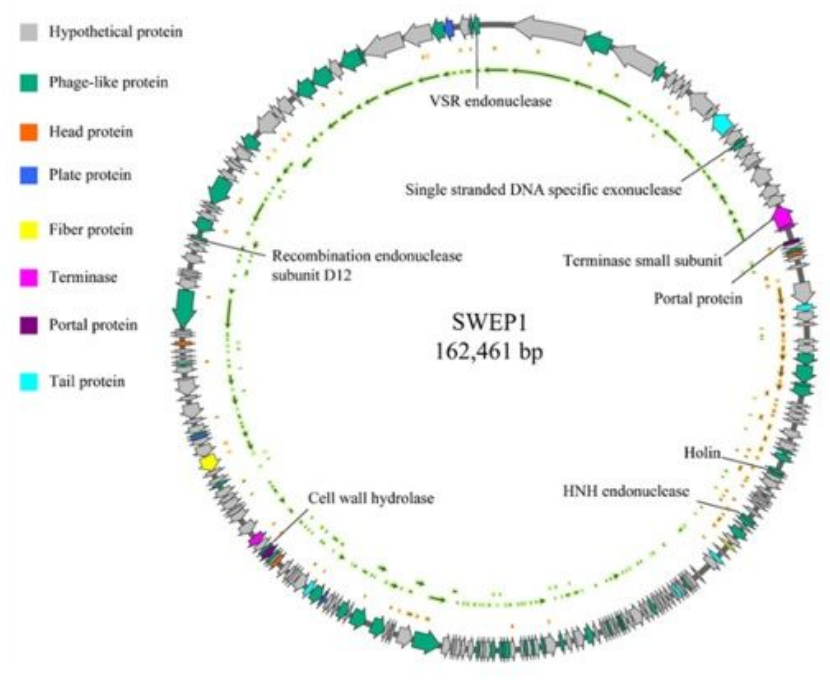

(b)

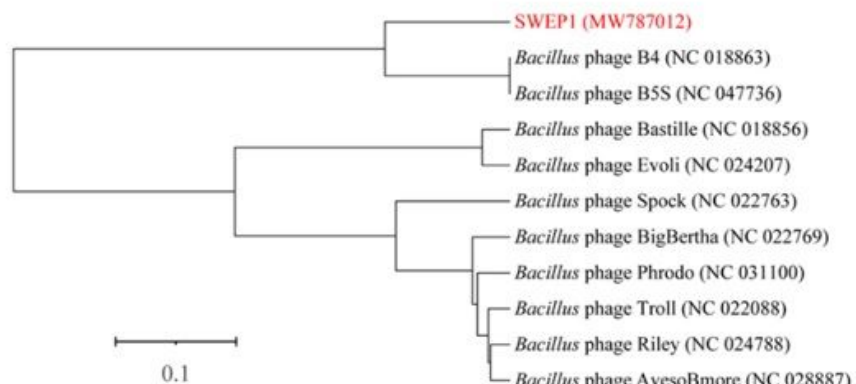

(c)

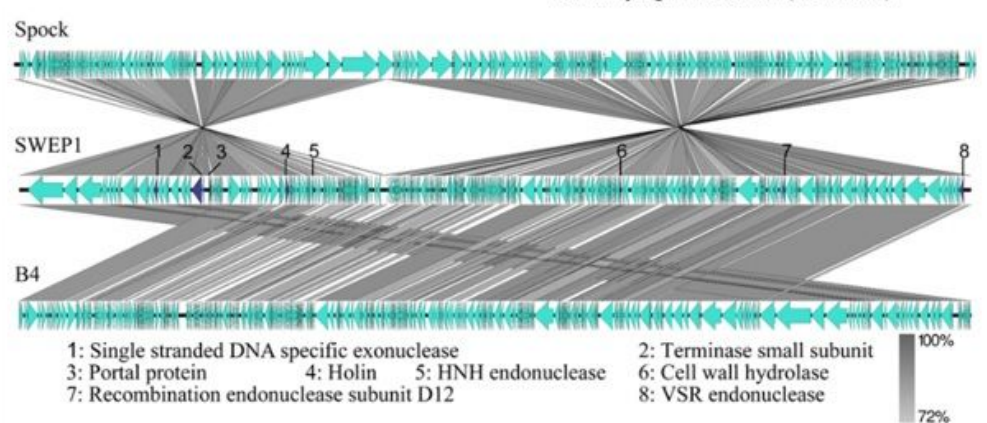

Figure 2

Genome features of phage. (a) SWEP1 annotated gene map. CDSs are represented by arrows with colors pointing in their transcriptional direction and putative functional roles. (b) Phylogenetic position of the SWEP1 genome. The similar genomes (top 10) in NCBI complete bacteriophages database were used to construct the phylogenetic tree. Scale bar is 0.1 substitutions per nucleotide position. (c) Genomic comparison of SWEP1, Bacillus phage B4, and phage Spock. Arrows represent CDSs and their transcriptional direction, labeled and purple arrows were assigned functions. Homologous regions in genomes are connected and shaded in grey according to their identity level. The visualization was done with Easyfig.

\section{Supplementary Files}

This is a list of supplementary files associated with this preprint. Click to download.

- NCBISubmissionofsequence.txt

- Supplementary.doc

- sequenceofSWEP1.fa 